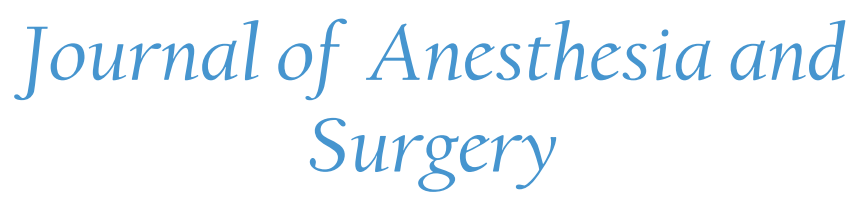

\title{
Changing Trends in Dental Procedural Sedation
}

\author{
Surjya Prasad Upadhyay ${ }^{*}$, Sudhakar Tellicherry ${ }^{1}$, Ulka Samant ${ }^{1}$, Arun Kumar Singh ${ }^{1}$, Malini Bhaga- \\ wati Rao ${ }^{2}$, Bhusan Jayade 3 , Piyush Mallick ${ }^{4}$
}

${ }^{1}$ Specialist Anesthesiology, NMC Hospital DIP, Dubai Investment Park, Dubai, UAE

${ }^{2}$ Consultant Anesthesiologist, BR medical Suit, Dubai Healthcare City, Dubai, UAE

${ }^{3}$ Specilaist Oral and Maxillo Facial Surgeon, NMC Hospital DIP, Dubai

${ }^{4}$ Anesthesiologist, Al Zahra Hospital, Sharjah, UAE

*Corresponding author: Dr. Surjya Prasad Upadhyay, MD, Specialist anaesthesiology, NMC Hospital DIP, Dubai Investment Park, Dubai, United Arab Emirates, Tel: 00971554078445; E-mail: run77in@yahoo.com

\begin{abstract}
Although most dental procedures are amenable under local anesthesia, but many a time, depending on the extent of procedure, anxiety or phobia, patient needs some degrees of sedation. Procedural sedation outside the operating room increases the risk of adverse events. Most commonly, near miss or critical events occur due to operator error or lack off rescue systems. Unfamiliar location, inadequate monitoring, insufficient or untrained staff and non-availability of emergency resuscitation equipment or medication in emergency situations are some of the major factors for adverse outcome. Proper monitoring; provision of readily available access to resuscitation facility and continuous presence of anesthesiologist have contributed to the decrease in death and disability caused by anesthesia related adverse events. Pre-procedural evaluation is done to screen patients for suitability for procedural sedation and assess the risk factors. Patients with full stomach, difficult airway or significant medical illness requiring more than mild sedation, alternative to procedural sedation should be consider. Clinician performing procedural sedation should have through knowledge of action, dose, side-effects, and antidote of commonly used sedative analgesics. Newer and innovative techniques have been evolved recently including transmucosal, transnasal, inhalational anesthetic, patient controlled sedation, target controlled sedation. All patients after dental procedural sedation should be monitored in a designated recovery area and should not be discharged until they meet all the discharge criteria and while sending home, proper written discharge instruction should be provided to all.
\end{abstract}

Received date: July 27, 2016

Accepted date: August 2, 2016

Published date: August 9, 2016

Citation: Upadhyay, S.P., et al. Changing Trends in Dental Procedural Sedation. (2016) J Anesth Surg 3(2): 159-168.

DOI: $10.15436 / 2377-1364.16 .049$

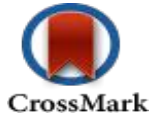

Keywords: Dental procedure; Procedural sedation; Outpatient sedation; Conscious sedation; Discharge criteria

\section{Introduction}

Most dental procedures may be done under Local Anesthesia but some patients (anxious, or requiring a more complex intervention) will require sedation. Advancements in dental technology have lead to the development of increasingly involved procedures, and many newer intravenous sedative medications have lead to a growing demand of procedural sedation for dental procedures being performed outside the operating theater, typically in the dental offices ${ }^{[1,2]}$. The recent years have seen an increase in out of OT sedation being administered in remote locations - in addition to the dental offices; we are giving sedation in the MRI Suite, gastroenterology offices, podiatry offices, urology suites, and in pain offices. All these remote locations offer similar requirements for patient sedation. We would like to consider a review of safety standards for equipment, personnel, and facilities that must be addressed while administering anesthesia in dental suites. These safety standards may be extended to include other remote sites of anesthesia administration.

In addition to inherent risks associated with the dental procedure, sedation provides an additional risk to the Procedure ${ }^{[3]}$. Stages of sedation is a continuum from fully conscious, minimal sedation, moderate, deep sedation to full unconsciousness which is

Copyrights: (C) 2016 Upadhyay, S.P. This is an Open access article distributed under the terms of Creative Commons Attribution 4.0 International License. 
consistent with general anesthesia where all protective reflexes get lost ${ }^{[4,5]}$. The likelihood of adverse events increases with increasing the depth of sedation. Procedural sedation can be defined as "a technique in which the use of a drug or drugs produces a state of depression of the central nervous system enabling treatment to be carried out, but during which verbal contact with the patient is maintained throughout the procedure." The drugs and techniques used to provide procedural sedation for dental treatment should carry a margin of safety wide enough to render loss of consciousness unlikely ${ }^{[6-8]}$.

Conscious sedation is popular and widely use term, but it is a misused or vague term as the level of sedation at per definition of conscious sedation by American Association of Physician (AAP) in 1985 is insufficient for most painful procedures, especially in children ${ }^{[9]}$. However, conscious sedation, when performed in a safe manner, is a valuable essential adjuvant to routine dental practice. The Anesthetic goals are to achieve a balanced plane of sedation where the patient may tolerate the dental Procedure while maintaining consciousness and verbal response to stimulation ${ }^{[10]}$. But there are no clear cut boundaries between moderate and deep sedation in all patients, particularly pediatric patients. The term sleep sedation was coined as an unarousable patient with retained breathing and airway reflexes ${ }^{[11]}$.

However, some childrens, developmentally delayed or mentally challenged adults, will require additional sedation / monitored anesthesia care with intravenous (IV) anesthetics and analgesic medications. Behavioral management in the form of explanation, counselling, and distraction is the main stay of anxiety management. If sedation deemed necessary, mild to moderate sedation in conjunction with proper local anesthesia is usually what is required ${ }^{[6,10,12]}$. With the exception of extensive oral maxillofacial surgery, the majority of dental cases involve minimal blood loss. Local anesthetics combined with post-procedure oral analgesics suffice for perioperative analgesia for the majority of dental cases $^{[13]}$.

The choice of personnel chosen to administer sedation should be defined in each hospital/dental policy document and is varied and maybe administered by the dentist doing the procedure, his nurse assisting him, the anesthesiologist working with the dentist, or an anesthesia nurse or assistant. The background in IV sedation and clinical experience with the possible side-effects of sedative medication makes the anesthesiologist a supremely qualified physician to administer sedation. An anesthesia nurse / assistant has similar background in sedation pharmacology and is a backup. If neither is available for sedation, the dentist or his nurse may administer sedation. However, it is best that the dentist or nurse attend some informative course on procedural sedation first that would provide some background on sedation first before administering it themselves. Regardless of who administers sedation, basic ASA monitors and standards of care for patients must be followed at all times. IV sedation causes depression of the respiratory and cardiac systems; cardiac arrest either secondary to respiratory arrest or primary is a very real possibility with IV sedation. It is therefore, advisable that all personnel involved with IV sedation have the advanced life support training as well to handle all possible complications of IV sedation to avoid mortality ${ }^{[10,14]}$.

\section{Risk and problem in dental sedation}

Dental procedural sedation involves administration of sedation/anesthesia outside the OT to patients undergoing painful or uncomfortable procedures. Major unique challenges with anesthesia outside OT include those related to patient, procedure and environment. Physician unfamiliar with the anesthesia outside operative room tends to underestimate the fact that patients undergoing procedure that require newer and advanced technological equipment are at higher risk ${ }^{[14]}$. Unfamiliar location, inadequate monitoring, insufficient or untrained staff and non-availability of emergency resuscitation equipment or medication in emergency situations places both anesthesiologist and patients at risk. Anesthesiologist must understand the nature of the procedure, invasiveness, positioning and duration for the procedure and will formulate the plan in advance in liaise with proceduralist including contingencies for emergencies and adverse outcomes. Nursing and technical staff involve in procedural sedation must be trained to assist or carry out immediate cardio-pulmonary resuscitation.

American Society of Anesthesiology (ASA) has provided minimal guidelines for anesthesia in the non operating room to improve the quality of patient care ${ }^{[15]}$.

Table 1: ASA Guidelines for Non-operating Room Anesthesia.

\begin{tabular}{|l|}
\hline Each location should have \\
\hline - Reliable source of oxygen adequate for the length of the procedure, with a backup supply \\
\hline - Adequate and reliable source of suction \\
\hline - Adequate and reliable system for scavenging waste anesthetic gases \\
\hline - Self-inflating hand resuscitator bag capable of administering > 90\% oxygen \\
\hline - Adequate anesthesia drugs, supplies, and equipment for the intended anesthesia care \\
\hline - Adequate monitoring equipment to allow adherence to the "Standards for Basic Anesthetic Monitoring" \\
\hline - Sufficient electrical outlets to satisfy anesthesia machine and monitoring equipment requirements \\
\hline Provision for adequate illumination \\
\hline - The patient, anesthesia machine, and monitoring equipment \\
\hline - Battery-powered illumination other than a laryngoscope immediately available \\
\hline Sufficient space \\
\hline - Accommodate necessary equipment and personnel \\
\hline - Allow expeditious access to the patient, anesthesia machine, and monitoring equipment \\
\hline \hline www.ommegaonline.org
\end{tabular}


Trends in Dental Procedural Sedation

\begin{tabular}{|l|}
\hline \hline Immediate availability of an emergency cart \\
\hline - Defibrillator, emergency drugs, and other equipment to provide cardiopulmonary resuscitation \\
\hline Staff \\
\hline - Trained anesthesiologist \\
\hline - Adequate staff trained to support the anesthesiologist \\
\hline Appropriate post-anesthetic management \\
\hline - Adequate number of trained staff \\
\hline - Appropriate equipment available to safely transport the patient to a post-anesthesia care unit \\
\hline
\end{tabular}

Complications in procedural sedation can be varies from mild difficulties to fatal one. (Table 2) Incidence of complications may be higher in non-operative room sedation than operative room ${ }^{[16]}$. When procedure sedation is conducted by non-anesthesiologist outside operative room, incidence of complications may not be different, but the rate of death and failure-to-rescue were greater when caring was not directed by anesthesiologists, demonstrating that 30-day morbidity and mortality were lower when anesthesiologists directed anesthesia care ${ }^{[17]}$. Procedural sedation outside the operating room increases the risk of adverse events. Most commonly, near miss or critical events that can occur due to operator error or lack off rescue systems. Continuous presence of anesthesiologist has contributed to the decrease in death and disability caused by anesthesia related perioperative events ${ }^{[18,19]}$.

Table 2: Complications of Non-operating Room Anesthesia

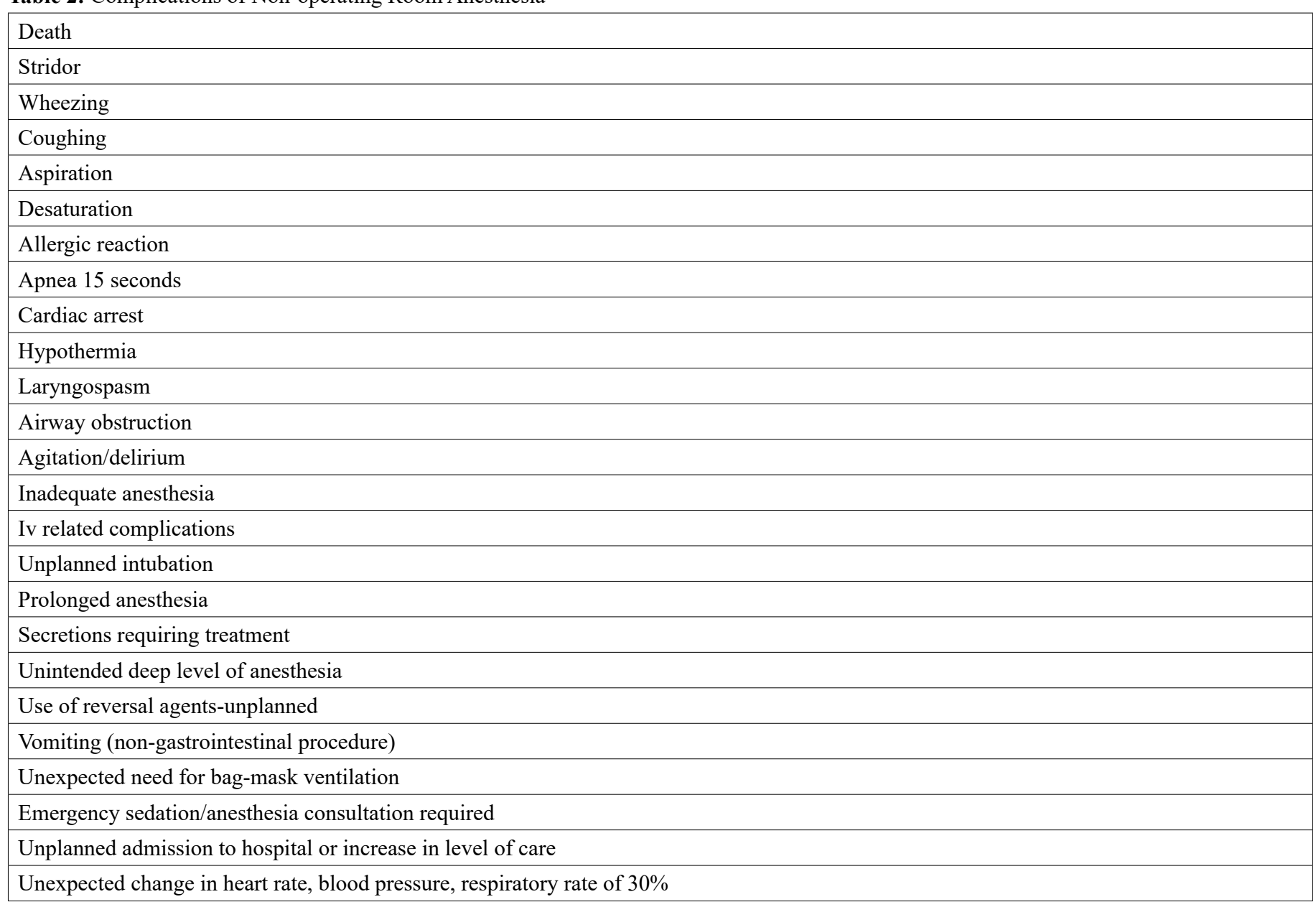

\section{Monitor and equipment}

The physical environment of dental room itself, with unfamiliar equipment and monitor location, restricted access to the airway pose unique challenges. Procedural sedation are in dental chair are not as frequent as in OT anesthesia, these locations are often equipped with older or malfunctioning monitors and resuscitation tools. Arrangement of things may not be in proper agronomic way such as oxygen supply, suction apparatus, and electrical outlet to name a few. Basic monitoring should be of same standard basic monitoring as in OT. Close claims frequently judged as substandard monitoring ${ }^{[16]}$. Physician providing sedation must be skilled and proficient in airway management and cardiovascular support and must be skillful to rescue patient from sedation deeper than intended. Continually evaluating and monitoring cardio-respiratory prior to, during and following the procedure is essential. There should be proper functioning resuscitation equipment such as anesthesia machine, equipment to secure airway, crash cart, and functioning suction apparatus. Most malpractice is caused not by the lack of skill of the anesthesiologist, but because of malfunctioning 
of resuscitation equipment due to negligent checkup, causing patient death. Periodic check and maintenance of equipment should be done by qualified personnel as per manufacturer guideline. Table 3 shows some equipment and monitoring requirement.

Table 3: Monitors and Equipment for Non-operating Room Anesthesia

\begin{tabular}{|l|l|l|l|}
\hline Physical plant & Environment & Monitors & Transport capability \\
\hline - Oxygen and backup & - Anesthesia machine & - Oximetry & - Oxygen delivery \\
\hline - Wall gases & - Oxygen delivery & - Capnography & - Oxygen tanks \\
\hline - Suction and evacuation & - Suction catheters & - Blood pressure & - Portable monitors \\
\hline - Visual access & - Intubation equipment & - Temperature & \\
\hline - Thermostatic control & - Intravenous pumps & & \\
\hline - Electrical outlets & & & \\
\hline
\end{tabular}

\section{Preparation of Patient before procedural sedation}

Pre-procedural assessment: Patients requiring sedation for dental procedure should be evaluated by a qualified anesthesiologist / seditionist according to institutional protocol. Ideally the evaluation should be done one or two days prior to intend procedure. A health care provider with current privilege to administer sedation-analgesia must conduct a pre-procedural evaluation. Detail history, cognitive functions, physical examination, airway assessment should be done on this pre-procedural assessment. Patient and, when appropriate, those with parental responsibility and careers require information to be provided in a way that can be easily understood before obtaining a valid written consent ${ }^{[5]}$.

Patient Selection: Procedural sedation in dentistry is an office or outpatient procedure, so only patient reasonably expected to meet the discharge criteria according to the institutional protocol should be selected for the dental sedation. Following group of patients are not suitable for dental sedation ${ }^{[6,8,20]}$.

1. Not able to co-operate because of

- Decrease level of consciousness- unable to understand because of reduced mental abilities.

- Unable to communicate- because of language barrier, deaf, mute etc.

- $\quad$ intoxication state- alcohol or substance abuse

- $\quad$ Patients with diagnosis of psychiatric illness

- $\quad$ organic brain syndrome- demented, senile state.

2. Patients with severe limitation of critical organ function- not suitable for day care procedure may need admission and observation for sometime after the procedure.

- $\quad$ Advanced COPD

- $\quad$ severe heart failure- not able to lie

- $\quad$ End stage renal failure

- $\quad$ Advanced liver failure

3. Abnormal airway- potential for airway problem

- morbidly obese patients with obstructive sleep apnea

- difficult airway on examination

- $\quad$ patient with significant symptomatic gastro-esophageal reflux

4. Difficult to handle on day care basis

- extreme of age

- $\quad$ Higher American society of Anesthesiologist Grading status (Above ASA-III).

- $\quad$ Prior adverse response to sedative- anesthetics

- $\quad$ pregnant patient

Presedation consent: Valid consent is necessary for all patients receiving sedation for any procedure and this must be confirmed in writing with a witness. It is a complex process; different laws and regulations apply at different ages in different countries. Practitioners must be aware and adherent to the laws that apply in their own country. The patient should be counseled about the risks of hypotension, hypoxia, bradycardia, cardiac dysrhythmias, respiratory depression, need for assisted ventilation, intubation, allergic reaction (swelling, rash) nausea, vomiting or achieving deeper sedation than intended.

Preprocedure fasting: Pre-sedation fasting is controversial and considered unnecessary by some authorities arguing that during minimal to moderate sedation, airway reflexes are maintained, but this does not consider the potential from inadvertent over-sedation and loss of protective airway reflexes ${ }^{[21,30]}$. 


\section{Techniques for sedation:}

Use of sedative drugs has been extensively researched and a number of techniques have been established and recognized throughout the world. There is a range of techniques available for procedural sedation. Whatever the technique is used, the selection of technique must be appropriate for the individual patient and procedure and not chosen simply for operator or sedationist convenience or at the insistence of a third party. Use of sedative medication should not negate the need for good communication and behavioral management. Adopting the principle of minimal intervention, the simplest and safest technique that is likely to be effective based on patient and clinical needs should be used.

Titration of sedative medication is very important in term of safety and effectiveness. Both over-sedation and under sedation have adverse effect on the patient and delivery of the effective treatment. As a general role single drugs are easy to titrate, combining multiple drugs may produce synergistic effects, have different timing for onset and peak effects and sometime unpredictable and difficult to titrate rendering safety margin narrowed, increasing the likelihood of overdose and cardio-respiratory depression. Another important issue is avoiding very strong sedative drugs with narrow therapeutic indices and reduced margin of safety potentially increasing the likelihood of adverse events. Use of such sedative or combination of drugs or techniques should only be used by experienced and skilled personal in their use in an approved setting, where there are clear clinical justifications. The techniques of sedation can be broadly divided into

- Oral sedation

- $\quad$ inhalation sedation with nitrous oxide

- Intravenous sedation with or without analgesics

- Newer and innovative techniques have been evolved recently including transmucosal, transnasal, inhalational anesthetic, patient controlled sedations, target controlled sedation, polypharmacy etc.

Inhalation of nitrous oxide with oxygen: Nitrous oxide is a time tested agent has been in use for over centuries and it has remained one of the most widely used anesthetic agent in either in dental or other surgical procedures ${ }^{[1]}$. It is the first line pharmacologic therapy for those requiring sedation/analgesia in addition to local infiltration and behavioral management ${ }^{[4,7,10]}$. When used alone with oxygen, it is impressively safe and excellent agent for providing anixolysis with mild analgesia for apprehensive dental patients. It is incapable of producing deep sedation or general anesthesia unless combined with other inhalational / intravenous sedative agent ${ }^{[22]}$. It is commonly delivered by appropriate nasal mask in upto $40-70 \%$ concentration through an oxygen-nitrous oxide blender that allow patient to inhaled while allowing surgeon to work in oral cavity ${ }^{[23]}$. As with of intravenous sedative agents, nitrous oxide should be administered only with standard protocol, the delivery device must employ specific safety features such as prevention of hypoxic mixture, use of pulse oximetry.

Intravenous sedation/analgesia: Various drugs available to provide procedural sedation depending on the invasivenss, duration of procedure, facilities for monitoring and resuscitation and expertise of the personnel involved in the sedation process. The greatest threat to the safety of intravenous sedation is airway compromise, loss of airway reflexes and risk of aspiration particularly in dental procedure and hemodynamic instability. To reduced the risk of cardio-respiratory compromise it is very essential to identify highrisk patient, appropriate selection of medications, adherence to dosing recommendation, appropriate intraprocedural monitoring and prompt intervention when adverse effects are recognized ${ }^{[2]}$. Most of the time Intravenous (IV) sedation is combined with nitrous oxide. Commonly used IV sedation either alone or combination of other IV sedative / analgesic are described as below.

Benzodiazepines (BZD): Benzodizepines provides number of favorable effects such as anxiolysis, amnesia, sedation and anticonvulsive effects. BZD binds to receptor sites in the Gamma Amino Butyric Acid (GABA) system, which facilitate the binding of GABA to its receptor and thus potentiates GABA-mediated chloride influx and resulting GABA-nergic actions. The BZD can have adverse effects on respiratory and hemodynamic function. Commonly used BZD for procedural sedations are midazolam and lorazepam.

Midazolam: Because of faster onset, less pain on injection, water soluble, more reliable amnesia and shorter duration of action makes it most commonly used BZD for procedural sedation in general. As BZD lack any analgesic action, it is often combined with opioid, generally fentanyl which resembled in pharmacokinetic profile (rapid onset, duration and offset). Use of fentanyl may not be necessary when a good local anesthetic infiltration is used. Of note, concomitant use of fentanyl and midazolam have synergistic action and reduces the requirement of each other ${ }^{[24]}$. Midazolam can be administered by various route including oral, nasal, buccal, transmucosal, intramuscular and intravenous ones. Midazolam can potentiate the respiratory depression action of opioid or other respiratory depressant ${ }^{[2,16]}$.

Lorazepam: Lorazepam is another BZD that can also be used for mild to moderate sedation; however, unlike midazolam, its onset is slower with prolonged duration of action( 6 - 8 hours) in comparison to 30 - 45 min of midazolam, making this drug unpopular for dental sedation.

Propofol: It is a potent intravenous anesthetic agent because of its unique pharmacologic profile of rapid onset, reliable sedation, rapid recovery and lack off active metabolite has accounted for its popularity in the arena of procedural sedation. Often used in sub-anaesthetic dosage to provide procedural sedation in various procedures outside the OT ${ }^{[25,26]}$. There is no analgesic action of propofol and is associated with number of side-effects such as pain on injection, rapid attainment and overshoot of depth of sedation 
than intended. Onset of action is very rapid with peak effects at $90-120 \mathrm{sec}$ with duration of action range from $5-10$ min depending on the dose ${ }^{[27-29]}$.

Ketamine: Ketamine causes profound analgesia with dissociative and amnestic action. It has strong analgesic action even at sub-anesthetic dose and with this dose it does not impair upper airway reflexes and thus allowing patient to breathe spontaneously and maintain the protective reflexes. However, though the reflexes may remain intact, but cannot be assumed to be protective ${ }^{[31]}$. Ketamine has dosed related side-effects notably increased muscle tone, may cause rigidity, increase in blood pressure and may results in a dissociative state and patient may not be able to speak or respond purposefully to verbal commands. With increasing doses it frequently causes emergence delirium described as vivid imaginary, hallucinations, confusion, excitement, irrational behavior, etc. which may last from 1 - 3 hours. Strayer RJ et al, 2008 and Newton A et al 2008 ${ }^{[32,33]}$, reported side-effects of ketamine include tachycardia, hypertension, laryngospasm, emergence delirium, hypersalivation, increased in intraocular, intragastric and intracranial pressure. The risk of laryngospasm may be greater in dental patients as the procedure itself can stimulate the oropharynx, presence of secretion and blood in orophrynx can stimulate laryngeal reflexes. Prevention of secretions by using antisialogogue and preventing blood from accumulating in the posterior pharynx and avoiding excessive stimulation of this region with suction device or other instruments in patients receiving ketamine for dental sedation ${ }^{[3]}$. Incidences of delirium and other side-effects can be minimized by limiting the dose of ketamine or using it in conjunctions with other sedative hypnotic. Commonly used combination medications with ketamine are midazolam, propofol and dexmedetomidine ${ }^{[34-37]}$.

Ketofol: Combination of ketamine and propofol is called ketofol, is a potentially alternative for procedural sedation, they are mostly combined together in same single syringe and are physically compatible for one hour at $23^{\circ} \mathrm{C}^{[38]}$. A number of studies have demonstrated that the varying proportion of combination in ketofol (1:1 to 1:10 in $\mathrm{mg}$ ) for sedation is safe and effective. The combination of the two agents appear to reduce side-effects of each medication used alone, and allows for a rapid recovery time ${ }^{[35,36,39-42]}$. The optimal ratio of ketamine to propofol in ketofol has not been defined yet. Higher ketamine proportion tends to cause more psychomimetic effects while higher propofol may cause more cardio-respiratory depression. Coulter et al. conducted a dose simulation study using 1:1 to 1:10 ketamine to propofol ratio combination for procedural sedation in paediatric and young adults (2 - $20 \mathrm{yrs})$. A ketamine-to-propofol ratio of 1:3 was the best combination for intermittent dosing. Ratio greater than 1:3 resulted delayed recovery. They suggested ketamine to propofol of 1:3 for boluses during short procedures (5-20 $\mathrm{min})$ and 1:4 ratio for short infusion as an alternative to intermittent boluses ${ }^{[42]}$.

Dexmedetomidine: Dexmedetomidine is a selective alpha-2 agnosit and has anxiolytic, sedative, analgesic and sympatholytic and reduces anesthetic requirements ${ }^{[43]}$. It has been used for procedural sedation in both pediatric and adult patients. It has many characteristics that make its use in procedural dentistry very appealing ${ }^{[44]}$. It is a sedative with no or minimal respiratory depression, antisialogogue, does not cause tachycardia or hypertension, and patients are easily arousable with clear mind ${ }^{[44-46]}$. Limited data on adult population undergoing dental procedural sedation with dexmedetomidine has shown it to efficacious and safe with sufficient analgesia, but limited amnesia and prolonged recovery time ${ }^{[47-50]}$. Although generally effective for sedation for noninvasive and short procedure, dexmedetomidine as sole agent has not been uniformly successful for invasive procedures ${ }^{[51-55]}$. It is slowly losing the popularity as sole agent for procedural sedation due to its slow onset, delayed recovery, minimal analgesia and limited amnesia. However, there is growing interest in combination of dexmedetomidine with ketamine because of anecdotal experience and few series of studies demonstrate the utility and rationale for combination therapy. When used together, dexmedetomidine may limit the tachycardia, hypertension, salivation, and emergence phenomena from ketamine, whereas, addition of ketamine speed up the sedation process and may prevent the bradycardia and hypotension that has been reported with dexmedetomidine ${ }^{[56-57]}$. Various regimens have been reported in the literature, the most common regimen appears to be the use of a bolus dose of both agents, dexmedetomidine $(1 \mu \mathrm{g} / \mathrm{kg})$ and ketamine $(1-2 \mathrm{mg} / \mathrm{kg})$, to initiate sedation followed by a dexmedetomidine infusion with supplemental bolus doses of ketamine as needed. Combination of ketamine and dexmedetomidine may be more appropriate for procedural sedation in patients with compromised respiratory or cardiac function. When compared with other agents used for procedural sedation, these two agents have limited effects on ventilator function when compared with other more commonly used agents ${ }^{[37,58,59]}$.

Opioids: Opoids mediate analgesia and dose-dependent sedation by acting on both $\mathrm{Mu}$ and Kappa opioid receptors. However, the degree of sedation using opioid as sole agent is less intense and unpredictable than that of other sedative-hypnotic. Moreover, high dose opioids are associated with respiratory depression and can occur with any opioid, which is dose-dependent and related to the potency of the chosen opioid ${ }^{[60]}$. In addition to sedation and respiratory depression, another respiratory effects is chest wall rigidity, which is more common with newer synthetic opioid especially when administered rapidly in large doses and perhaps more common in younger patients ${ }^{[61,62]}$. Longer acting opioid such as morphine, pethidine are certainly not the agent of choice for procedural sedation because of slower onset and longer duration of action. Shorter acting opioid like fentanyl, alfentanyl sufentanil are often used as analgesic component along with other sedative agent for painful procedural sedation ${ }^{[63]}$. The role of opioid in procedural sedation in dentistry is questionable as the analgesic effect is trivial in most cases because most patients receive local anesthesia. Opioid may be considered in long and difficult dental procedure where local anesthesia may be ineffective because of local infection or invasiveness of the procedure. Opioid are also helpful in reliving discomfort associated with prolonged motionless sitting with mouth wide open. When used with other sedative agent it has synergistic actions and pre-emptive use of opioid may diminish the discomfort associated with local anesthetic injection. Unlike the analgesic effects of opioids which follow a fairly consistent dose-response, the 
$\overline{\text { sedation and other side-effects do not always follow this pattern and higher doses invariably lead to unpleasant side-effects like as }}$ nausea and vomiting ${ }^{[64]}$.

\section{Factor influencing efficacy and safety of procedural sedation}

There are numbers of factors which should be considered before giving any sedative-analgesics. Range of patient response to any particular sedative or an analgesic may vary from three to fivefold. Because of this variation in response, clinician attempting to induce moderate sedation with a single bolus dose may only produce mild sedation in some and might lend up with deep sedation in others. It is better to start with minimal dose and then titrate the drug by incremental dosing based on pharmacodynamic properties of the agent until the desired level of sedation is achieved.

Patients with known or anticipated difficult airway especially those with difficult to ventilation- moderate to deep sedation should be avoided as there is a risk of loss of airway. Patients with major co-morbid condition and in elderly; a more conservative approach or modification of procedural sedation technique can be done such as giving lower rate of drug administration and slow titration.

Patients with extreme of age: Limited organ reserve- risk of over-sedation ${ }^{[65-67]}$.

Table 4: Patient Factors Affecting Response to Sedation

\begin{tabular}{|l|l|}
\hline Morbidity & Organ System Abnormalities \\
\hline Difficult airways & $\begin{array}{l}\text { Sleep apnea; obesity; short neck; reduced mouth opening; large tongue; anatomical abnormal- } \\
\text { ities }\end{array}$ \\
\hline Risks of aspiration & $\begin{array}{l}\text { Acute upper gastrointestinal bleeding; gastric outlet obstruction; delayed gastric emptying; } \\
\text { achalasia }\end{array}$ \\
\hline $\begin{array}{l}\text { Reduced tolerance / paradoxical reactions } \\
\text { to standard sedatives }\end{array}$ & $\begin{array}{l}\text { Tobacco, alcohol, or substance abuse; previous adverse experience with sedation; neuropsychi- } \\
\text { atric disorder; allergies; drug interactions; extreme of age }\end{array}$ \\
\hline
\end{tabular}

Paediatric consideration: The choice and selection of agent for pediatric procedural sedation differ slightly. Traditionally, oral sedation has been the mainstay of pediatric dental sedation. Because of behavioral constraints, sedation by bolus oral administration is well tolerated and routine; but sedation depth with oral medication is difficult to predict and impossible to titrate the dose ${ }^{[13,68,69]}$. However, with development of technology and better pharmacologic profile of modern drugs in new ways with high success, newer alternate routes has been proposed and investigated such as transmucosal (intranasal, buccal, sublingual) which has much less discomfort than intravenous route with faster and predictable action than oral sedation ${ }^{[6]}$. Midazolam is the most frequently used sedative agent in pediatric and can be administered via various route (oral, nasal, rectal, intramuscular, intravenous). It provides excellent anxiolytic and amnesia. It can be safely combined with intranasal fentanyl. Another agent frequently used in pediatric is ketamine which can also be given through various route ${ }^{[69-71]}$. Propofol and dexmedetomidine is also being increasingly used in paediatric procedural sedation. Dexmedetomidine can also be administered through buccal, intranasal or oral route $\mathrm{e}^{[72]}$.

Unlike adult patients, pediatric patients require careful preoperative evaluation and intraoperative monitoring during the procedural sedation as most of the patients in this group require moderate to deeper level of sedation which may compromise the cardio-respiratory mechanics. Many pediatric patients may actually lend up into general anesthesia; pediatric patients should be prepared and proper fasting guideline to be adhered as if they are going to have full general anesthesia.

Pregnancy: Sedation is generally avoided in pregnant patients, in exceptional cases such patient can be taken up for procedural sedation with proper precaution ${ }^{[33]}$.

- $\quad$ Gastro prophylaxis: pre-procedural administration of prokinetic (metoclopramide) to reduce gastric content and decreased stomach acidity (H2 antagonist, proton pump inhibitor, sodium citrate) may reduce the risk of vomiting and aspiration.

- $\quad$ Pre-procedural hydration and left lateral displacement of uterus- to reduce the risk of hypotension due to aorto caval compression. Fetal monitoring is not required, but should be considered for women in the third trimester.

- $\quad$ Oxygen supplementation to counteract the risk of sedation-related maternal desaturation.

- Potential teratogenic drugs to be avoided.

Postprocedural care: As sedation is stimulus-dependent, at the end of the procedure as there is no stimulus, patient is likely to become more sedated than during the procedure itself. This can lead to hypoventilation and hypoxia in susceptible patients (extreme of age, patients with cardio-respiratory disease). After the completion of dental procedure, it is important to keep monitoring and recording the vital sign, medications, fluid until the patient responds to verbal or tactile stimulation.

Discharge Criteria: Dental procedural sedation is mostly done in office set up in dental chair and patients are often discharge home directly from the post-recovery area. To ensure that these patients are discharge home safely and efficiently, they must meet discharge criteria before being sent to home. It is not uncommon for patients to experience mild symptoms, such as nausea, lightheadedness, fatigue, or unsteadiness for up to 24 hours. Serious adverse events, such as hypoxia, rarely occur after discharge. This should be made clear to the patient.

In general, discharge criteria should include ${ }^{[74]}$.

- Protective reflexes are intact and the patient exhibits no signs of respiratory distress 
- Vitals sign should be within $15 \%$ of preprocedural value.

- $\quad$ Consciousness - patient should be awake, alert and responds to commands appropriate to age.

- $\quad$ Able to sit upright or ambulate with assistance without hypotension.

- $\quad$ No pain or minimal pain that can be controlled with simple analgesics.

- $\quad$ No active bleeding. Should not be actively vomiting and nausea if any should be mild.

- $\quad$ A minimum of 30 minutes has elapsed since the end of the procedure; and

- $\quad$ The patient should be accompanied by a responsible adult.

- $\quad$ Post-discharge instruction- should be given in writing in clear and understandable language by the patient or caretaker.

\section{Summary and Recommendations}

Procedural sedation can be defined as "a technique in which the use of a drug or drugs produce a state of depression of the central nervous system enabling treatment to be carried out, but during which verbal contact with the patient is maintained throughout the procedure." The drugs and techniques used to provide procedural sedation for dental treatment should carry a margin of safety wide enough to render loss of consciousness unlikely.

Procedural sedation outside the OT increases the risk of adverse events. Most commonly, near miss or critical events occur due to operator error or lack off rescue systems. Unfamiliar location, inadequate monitoring, insufficient or untrained staff and non-availability of emergency resuscitation equipment or medication in emergency situations are some of the major factors for adverse outcome.

Hospital /dental setup should have a clear policy for sedation protocol. The personnel, equipment, monitoring standard and post-procedure care and discharge should be clearly defined in such policy statement. All the personnel with privilege to administer sedation for procedural dentistry must have skill and training in advanced life support measures. There should be proper functioning resuscitation equipment such as anesthesia machine, equipment to secure airway, crash cart, functioning suction apparatus.

Meticulous pre-procedural evaluation, appropriate patient selection, adherence to fasting guideline, informed consent and formulate a plan of action are mainstay for success of procedural sedation.

Dental procedural sedation involves counseling, behavioral management, use of local anesthetic and pharmacologic analgesic- sedative are all central components of care of patients who are frequently anxious for the dental procedure.

Adopting the principle of minimal intervention, the simplest and safest technique that is likely to be effective based on patient and clinical needs should be used.

No single drug is ideal for all situations. Choice of technique or sedation depend patient type, invasiveness and length of the procedure and presence of co-morbid conditions. Counseling to patient (and the family) behavioral management and use of local anesthetic suffice in most dental procedure.

For apprehensive patient oral sedation in the form of short acting benzodiazepine (midazolam, lorazepam) night before and morning of the procedure reduces anxiety related to the procedural phobia.

Nitrous oxide is the first line therapy for those requiring sedation/analgesia in addition to local infiltration and behavioral management.

Patients with invasive / prolonged procedure or uncooperative (children/ mentally challenge adult) require intravenous (IV) sedative /analgesic in addition to local anesthetics and nitrous oxide; commonly used IV agents are midazolam, propofol, fentanyl, alfentanyl, ketamine, dexmedetomidine either as sole agent or in varying combination. The greatest threat to the safety of intravenous sedation is airway compromise, loss of airway reflexes and risk of aspiration particularly in dental procedure and hemodynamic instability. Full monitoring and resuscitative facility must be there before using IV sedative analgesics and it should only be administered by personnel skill in airway and life support measure

Newer and innovative techniques are being evolving such as transmucosal, transnasal, inhalational anesthetic, patient controlled sedation, target controlled sedation, polypharmacy, etc. they have been validated as safe and effective for diverse procedural sedation including dental procedures.

All patients after procedural sedation should be monitored in a designated recovery area and should not be discharged until they meet all the discharge criteria and while sending home, proper written discharge instruction should be provided to all. 


\section{References}

1. Krauss, B., Green, S.M. Procedural sedation and analgesia in children. (2006) Lancet 367(9512): 766-780.

2. Tobias, J.D., Leder, M. Procedural Sedation: A review of sedative agents, monitoring and management of complication. (2011) Saudi J Anesth 5(4): 395-410.

3. Goodwin, S.A., Caro, D.A., American College of Emergency Physicians, et al. Clinical policy: Procedural sedation and analgesia in the emergency department. (2005) Ann Emerg Med 45(2): 177-196.

4. Academy of Medical Royal Colleges. Safe Sedation Practice for health care Procedures. (2013) AoMRC, London.

5. American Society of Anesthesiologists. Continuum of depth of sedation: definition of general anesthesia and levels of sedation/analgesia. (2004)

6. Scottish Dental Clinical Effectiveness Programme. Conscious Sedation in Dentistry Dental Clinical Guidance. 2nd Edn. (2012) Dundee: SDCEP.

7. National Institute for Health and Clinical Excellence. Sedation in Children and Young People. (2010) London: NICE.

8. Academy of Medical Royal Colleges. Implementing and Ensuring Safe Sedation Practice for Healthcare Procedures in Adults. (2001) London: AoMRC.

9. Guidelines for the elective use of conscious sedation, deep sedation, and general anaesthesia in paediatric patients. (1985) Pediatr Dent 76(2): 334.

10. Standards for Conscious Sedation in the Provision of Dental Care. Report of the Intercollegiate Advisory Committee for Sedation in Dentistry. (2015) The dental faculties of the royal colleges of surgeons and the Royal College of Anaesthetists.

11. Sury, M.R., Hatch, D.J., Deeley, T., et al. Development of a nurse-led sedation service for paediatric magnetic resonance imaging. (1999) Lancet 353(9165): 1667-1671.

12. Sheta, S.A. Procedural sedation analgesia. (2010) Saudi J Anaesth 4(1): 11-16.

13. American Academy of Pediatrics and the American Academy on Pediatric Dentistry. Guideline for monitoring and management of pediatric patients during and after sedation for diagnostic and therapeutic procedures. (2006) Pediatr Dent 37(6): 211-227.

14. Youn, A.M., Ko, Y-K., Kim, Y-H. Anesthesia and sedation outside of the operating room. (2015) Korean J Anesthesiol 68(4): $323-331$.

15. Statement on non operating room anesthetizing location. (2010) American Society of Anesthesiologists.

16. Robbertze, R., Posner, K.L., Domino, K.B. Closed claims review of anesthesia for procedures outside the operating room. (2006) Curr Opin Anesthesiol 19(4): 436-442.

17. Abenstein, J.P., Warner, M.A. Anesthesia providers, patient outcomes, and costs. (1996) Anesth Analg 82(6): 1273-1283.

18. Melloni, C. Morbidity and mortality related to anesthesia outside the operating room. (2005) Minerva Anestesiol 71(6): 325-334.

19. Bell, C., Sequeira, P.M. Nonoperating room anesthesia for children. (2005) Curr Opin Anesthesiol 18(3): 271-276.

20. Jackson, D.L., Johnson, B.S. Conscious sedation for dentistry: risk management and patient selection. (2002) Dent Clin North Am 46(4): 767780.

21. Murphy, P.J., Erskine, R., Langton, J.A. The effect of intravenously administered diazepam, midazolam and flumazenil on the sensitivity of upper airway reflexes. (1994) Anaesthesia 49(2): 105-110.

22. Browne, D.R., Rochford, J., O'Connell, U., et al. The incidence of postoperative atelectasis in the dependent lung following thoracotomy: the value of added nitrogen. (1970) Br J Anaesth 42(4): 340-346.

23. Emmanouil, D.E., Quock, R.M. Advances in understanding the actions of nitrous oxide. (2007) Anesth Prog 54(1): 9-18.

24. Ben-Shlomo, I., Abd-El-Khalim, H., Ezry, J., et al. Midazolam Acts synergistically with Fentanyl for induction of Anaesthesia. (1990) Br J Anesth 64(1): 45-47.

25. Harris, C.E., Grounds, R.M., Murray, A.M., et al. Propofol for long-term sedation in the intensive care unit. A comparison with papaveretum and midazolam. (1990) Anaesthesia 45(5): 366-372.

26. Beller, J.P., Pottecher, T., Lugnier, A., et al. Prolonged sedation with propofol in ICU patients: Recovery and blood concentration changes during periodic interruption in infusion. (1988) Br J Anaesth 61: 583-588.

27. Green, S.M., Krauss, B. Propofol in emergency medicine: pushing the sedation frontier. (2003) Ann Emerg Med 42(6): $792-797$.

28. Black, E., Campbell, S.G., Magee, K., et al. Propofol for Procedural Sedation in the Emergency Department: A Qualitative Systematic Review. (2013) Ann Pharmacother 47(6): 856-868.

29. Newstead, B., Bradburn, S., Appelboam, A., et al. Propofol for adult procedural sedation in a UK emergency department: safety profile in 1008 cases. (2013) Br J Anaesth 111(4): 651-655.

30. Apfelbaum, J.F., Caplan ,R.A., American Society of Anesthesiologists Committee, et al. Practice Guidelines for Preoperative Fasting and Use of Pharmacologic Agents to reduce the risk of Pulmonary Aspiration: Application to Healthy Patients Undergoing Elective Procedures. (2011) Anesthesiology 114(3): 495-511.

31. Green, S.M., Roback, M.G., Kennedy, R.M., et al. Clinical practice guideline for emergency department ketamine dissociative sedation. (2011) Ann Emerg Med 57(5): 449-461.

32. Strayer, R.J., Nelson, L.S. Adverse events associated with ketamine for procedural sedation in adults. (2008) Am J Emerg Med 26(9): 985-1028. 33. Newton, A., Fitton, L. Intravenous ketamine for adult procedural sedation in the emergency department: a prospective cohort study. (2008) Emerg Med J 25(8): 498-501.

34. Sener, S., Eken, C., Schultz, C.H., et al. Ketamine with and without midazolam for emergency department sedation in adults: a randomized controlled trial. (2011) Ann Emerg Med 57(2): 109-114.

35. Andolfatto, G., Willman, E. A Prospective Case Series of Single-syringe Ketamine-Propofol (Ketofol) for Emergency Department Procedural Sedation and Analgesia in Adults. (2011) Acad Emerg Med 18(3): 237-245.

36. Miner, J.R., Moore, J.C., Austad, E.J., et al. Randomized, Double-Blinded, Clinical Trial of Propofol, 1:1 Propofol/Ketamine, and 4:1 Propofol/ Ketamine for Deep Procedural Sedation in the Emergency Department. (2014) Ann Emerg Med 65(5): 479-488.

37. Tobias, J.D. Dexmedetomidine and ketamine: an effective alternative for procedural sedation? (2012) Pediatr Crit Carre Med 13(4): $423-427$. 38. Trissl, L.A., Gilbert, D.L., Martinez, J.F. Compatibility of propofol injectable emulsion with selected drugs during simulated Y-site administration. (1997) Am J Health-Syst Pharm 54(11): 1287-1292.

39. Aouad, M.T., Moussa, A.R., Dagher, C.M. Addition of ketamine to propofol for initiation of procedural anesthesia in children reduces propofol consumption and preserves hemodynamic stability. (2008) Acta Anaesthesiol Scand 52(4): 561-565. 
40. Badrinath, S., Avramov, M.N., Shadrick, M., et al. The use of a ketamine-propofol combination during monitored anesthesia care. (2000) Anesth Analg 90(4): 858-862.

41. Daabiss, M., Elsherbiny, M., Alotibi, R. Assessment of different concentration of Ketofol in procedural operation. (2009) BJMP 2(1): 27-31.

42. Coulter, F.L., Hannam, J.A., Anderson, B.J. Ketofol dosing simulations for procedural sedation. (2014) Pediatr Emerg Care 30(9): 621-630.

43. Gerlach, A.T., Dasta, J.F. Dexmedetomidine: an updated review. (2007) Ann Pharmacother 41(2): 245-252.

44. Devasya, A., Sarpangala, M. Dexmedetomidine: A Review of a Newer Sedative in Dentistry. (2015) J Clin Pediatr Dent 39(5): $401-409$.

45. Ebert, T.J., Hall, J.E., Barney, J.A., et al. The effects of increasing plasma concentrations of dexmedetomidine in humans. (2000) Anesthesiology 93(2): 382-394.

46. Dyck, J.B., Maze, M., Haack, C., et al. Computer-controlled infusion of intravenous dexmedetomidine hydrochloride in adult human volunteers. (1993) Anesthesiology 78(5): 821-828.

47. Ustun, Y., Gunduz, M., Erdogan, O., et al. Dexmedetomidine versus midazolam in outpatient third molar surgery. (2006) J Oral Maxillofac Surg 64(9): 1353-1358.

48. Cheung, C.W., Ying, C.L., Chiu, W.K., et al. A comparison of dexmedetomidine and midazolam for sedation in third molar surgery. (2007) Anaesthesia 62(11): 1132-1138.

49. Taniyama, K., Oda, H., Okawa, K., et al. Psychosedation with dexmedetomidine hydrochloride during minor oral surgery. (2009) Anesth Prog 56(3): 75-80.

50. Makary, L., Vornik, V., Finn, R., et al. Prolonged recovery associated with dexmedetomidine when used as a sole sedative agent in office-based oral and maxillofacial surgery procedures. (2010) J Oral Maxillofac Surg 68(2): 386-391.

51. Munro, H.M., Tirotta, C.F., Felix, D.E., et al. Initial experience with dexmedetomidine for diagnostic and interventional cardiac catheterization in children. (2007) Pediatr Anesth 17(2): 109-112.

52. Young, E.T. Dexmedetomidine sedation in a pediatric cardiac patient scheduled for MRI. (2005) Can J Anaesth 52(7): 730-732.

53. Heard, C.M.B., Joshi, P., Johnson, K. Dexmedetomidine for pediatric sedation: A review of a series of cases. (2007) Pediatr Anesth 17(9): 888-892.

54. Jalowiecki, P., Rudner, R., Gonciarz, M., et al. Sole use of dexmedetomidine has limited utility for conscious sedation during outpatient colonoscopy. (2005) Anesthesiology 103(2): 269-273.

55. Alhashemi, J.A. Dexmedetomidine vs. midazolam for monitored anaesthesia care during cataract surgery. (2006) Br J Anaesth 96(6): $722-726$. 56. Levänen, J., Mäkelä, M.L., Scheinin, H. Dexmedetomidine premedication attenuates ketamine-induced cardiostimulatory effects and postanesthetic delirium. (1995) Anesthesiology 82(5): 1117-1125.

57. Dilek, O., Yasemin, G., Atci, M. Preliminary experience with Dexmedetomidine in Neonatal Anesthesia. (2011) J Anesth Clin Pharm 27(1): $17-22$.

58. Mahmoud, M., Tyler, T., Sadhasivam, S. Dexmedetomidine and ketamine for large anterior mediastinal mass biopsy. (2008) Pediatr Anesth 18(10): 1011-1013.

59. Luscri, N., Tobias, J.D. Monitored anesthesia care with a combination of ketamine and dexmedetomidine during magnetic resonance imaging in three children with trisomy 21 and obstructive sleep apnea. (2006) Pediatr Anesth 16(7): 782-786.

60. Satoh, M., Minami, M. Molecular pharmacology of the opioid receptors. (1995) Pharmacol Ther 68(3): 343-364.

61. Fahnenstich, H., Steffan, J., Kau, N., et al. Fentanyl-induced chest wall rigidity and laryngospasm in preterm and term infants. (2002) Crit Care Med 28(3): 835-839.

62. Muller, P., Vogtmann, C. Three cases with different presentation of fentanyl-induced muscle rigidity-a rare problem in intensive care of neonates. (2000) Am J Perinatol 17(1): 23-26.

63. Tobias, J.D., Leder, M. Procedural sedation: A review of sedative agents, monitoring, and management of complications. (2011) Saudi J Anaesth 5(4): 395-410.

64. Becker, D.E. Pharmacodynamic Considerations for Moderate and Deep Sedation. (2012) Anesth Prog 59(1): $28-42$.

65. Cepeda, M.S., Farrar, J.T., Baumgarten, M., et al. Side effects of opioids during short-term administration: effect of age, gender, and race. (2003) Clin Pharmacol Ther 74(2): 102-112.

66. Miner, J.R., Burton, J.H. Clinical practice advisory: Emergency department procedural sedation with propofol. (2007) Ann Emerg Med 50(2): 182-187.

67. Miller, M.A., Levy, P., Patel, M.M. Procedural sedation and analgesia in the emergency department: what are the risks? (2005) Emerg Med Clin North Am 23(2): 551-572.

68. Nelson, T., Nelson, G. The role of sedation in contemporary pediatric dentistry. (2013) Dent Clin North Am 57(1): $145-161$.

69. Nelson, T.M., Xu, Z. Pediatric dental sedation: challenges and opportunities. (2015) Clin Cosmet Investig Dent 7: 97-106.

70. Gazal, G., Fareed, W.M., Zafar, M.S. Pain and anxiety management for pediatric dental procedures using various combinations of sedative drugs: A review. (2016) Saudi Pharm J 24(4): 379-385.

71. Kost, S., Roy, A. Procedural Sedation and Analgesia in the Pediatric Emergency Department: A Review of Sedative Pharmacology. (2010) Clin Pediatr Emerg Med 11(4): 233-243.

72. McMorrow, S.P., Abramo, T.J. Dexmedetomidine sedation: uses in pediatric procedural sedation outside the operating room. (2012) Pediatr Emerg Care 28(3): 292-296.

73. Neuman, G., Koren, G. Safety of procedural sedation in pregnancy. (2013) J Obster Gynaecol Can 35(2): 168-173.

74. Newman, D.H., Azer, M.M., Pitetti, R.D., et al. When is a patient safe for discharge after procedural sedation? The timing of adverse effect events in 1367 pediatric procedural sedations. (2003) Ann Emerg Med 42(5): 627-635.

Ommega Online Publishers

Journal Title: Journal of Anesthesia and Surgery (JAS)

Journal Short Name: J Anesth Surg
Journal ISSN: 2377-1364

E-mail: anestheisa@ommegaonline.com

Website: www.ommegaonline.org 